\title{
EVOLUCIÓN DEL PAISAJE Y ACTIVIDAD HUMANA EN EL ÁREA DE MONTE PENIDE (REDONDELA, PONTEVEDRA): UNA APROXIMACIÓN METODOLÓGICA
}

\author{
LANDSCAPE CHANGES AND HUMAN ACTIVITY IN MONTE PENIDE (REDONDELA, \\ PONTEVEDRA): A METHODOLOGICAL APPROACH
}

ANTONIO MARTÍNEZ CORTIZAS (*)

RAMÓN FÁBREGAS VALCARCE $(* *)$

SUSANA FRANCO MASIDE $(*)$

\section{RESUMEN}

Hemos prospectado dos áreas con manifestaciones de arte rupestre en Monte Penide, descubriendo en su cercanía concentraciones de material cerámico y lítico de cronología semejante. Uno de los lugares examinados (Coto da Fenteira) fue objeto de un análisis edafológico sobre un corte abierto por obras recientes. La combinación de estudios sobre elementos traza ( $\mathrm{Ti}, \mathrm{Zr}, \mathrm{Hg}$ o $\mathrm{Br}$ ) y de dataciones $\mathrm{C}-14$ ha permitido definir un suelo policíclico que se extiende a lo largo del Holoceno y en el que se diferencian dos grandes episodios erosivos vinculados a la acción antrópica: uno a partir de la primera mitad del III milenioAC y otro menos violento desde inicios del II milenio, coincidentes grosso modo con la expansión del sistema agropastoril durante el Calcolítico y su continuación a lo largo del Bronce inicial/medio regional, desmintiendo así la noción de una crisis socioeconómica durante el segundo período y apoyando, en cambio, la hipótesis de una incidencia progresiva de los grupos humanos sobre el medio hasta bien entrada la Edad del Hierro.

\section{ABSTRACT}

We have surveyed two areas with open air rock art in Monte Penide, finding in the process several artefact sca-

(*) Universidade de Santiago de Compostela. Dpto. de Edafoloxía e Química Agrícola. Facultade de Bioloxía. 15706 Santiago de Compostela. Correo electrónico: edantxon@usc.es.

(**) Universidade de Santiago de Compostela. Dpto. de Historia 1. Facultade de Xeografía e Historia. 15704 Santiago de Compostela. Correo electrónico: phfabreg@usc.es.

El artículo fue remitido en su versión final el 9-III-2000. tters near the petroglyphs of roughly the same age. Also, we have undertaken a soil analysis of a section already exposed in one of those places (Coto da Fenteira), looking for occurrences of enrichment in certain trace elements (Ti, $\mathrm{Zr}$, $\mathrm{Hg}$ or $\mathrm{Br}$ ) along the sequence. As a result of this studies in combination with $14 \mathrm{C}$ dating of charcoal and organic matter, we have been able to define two main erosive episodes, resulting from human activity: one in the first half of the third millenium BC and a second, less violent one, from the start of the second millenium $B C$. These episodes have to do mostly with the spread of a farming economy during the Chalcolithic and its consolidation along the Earlier Bronze Age, thus rejecting the notion of a socioeconomic crisis during the latter and showing, instead, a progressive interference with the environment by human groups up to the second Iron Age.

Palabras clave: Paleoambiente. Edafología. Elementos traza. Petroglifos galaicos. Edad del Bronce. Península Ibérica.

Key words: Palaeoenvironment. Soil science. Trace elements. Galician petroglyphs. Bronze Age. Iberian Peninsula.

\section{INTRODUCCIÓN}

Monte Penide (Redondela) es una zona que constituye un pequeño promontorio por encima de los $350 \mathrm{~m}$ de altitud s.n.m., dispuesto entre la costa meridional de la ría de Vigo y el río Maceiras (Fig. 1) y su parte superior está integrada por una llanada rodeada por pequeñas elevaciones. Hacia el 


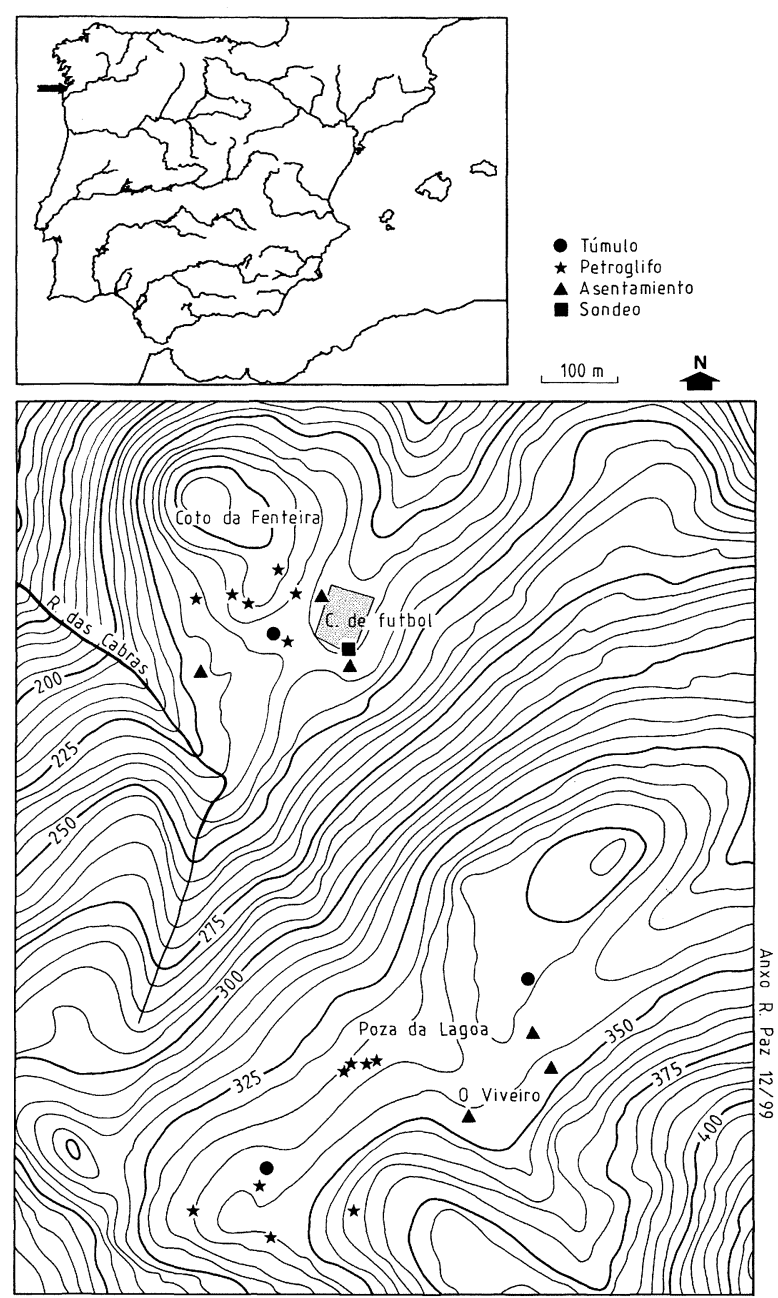

Fig. 1. Localización del área de Monte Penide (Redondela, Pontevedra).

NNO se produce un descenso hacia el litoral mediante una sucesión de bruscas pendientes alternadas con rellanos en los que por razones topográficas tiene lugar una acumulación de humedad, provocando incluso encharcamientos o la aparición de manantiales en la actualidad. Es en este último sector donde llevamos a cabo nuestras exploraciones y concretamente en los replanos denominados Coto da Fenteira y Poza da Lagoa, a una altura media de 235 m y 340 m s.n.m., respectivamente. El objetivo primario de la investigación de campo consistía en el análisis detallado del emplazamiento y características de los petroglifos allí existentes y su eventual relación con otra clase de yacimientos, habitacionales o funerarios. Subsidiariamente y a la vista de los hallazgos efectuados y de la existencia en Coto da Fenteira de un gran corte -provocado por la construcción de un campo de fútbol-, nos plan- teamos la realización de análisis paleobotánicos (en curso) y edafológicos (de la autoría de A.M.C. y S.F.M.), con el fin de responder en la medida de lo posible a dos cuestiones: la incidencia de las actividades humanas sobre el entorno y, por otra parte, verificar la existencia de la alegada recesión socioeconómica del II milenio. No disponiendo todavía de los resultados de los análisis polínicos, serán las valiosas informaciones proporcionadas por los estudios pedológicos, confrontadas y complementadas con los datos arqueológicos, las que constituirán el núcleo del presente artículo.

\section{La evidencia arqueológica y su problemática}

El área llana de Monte Penide destaca por la abundancia de restos arqueológicos encuadrables en la Prehistoria reciente, entre los que se distinguen la gran necrópolis tumular y, en posición más periférica, algunas estaciones rupestres, con motivos mayoritariamente geométricos, así como sendas dispersiones cerámicas que podrían corresponder a lugares de habitación del III/II milenios AC. Los lugares prospectados se localizan en situación marginal respecto al planalto penidés, pero en cambio controlan una serie de rutas que comunican la zona anterior con la llanura costera. El área de Poza da Lagoa exhibe el mayor número (11) y variedad de petroglifos, entre ellos una superficie con grabados de puñales y alabardas, además de sendos túmulos y una dispersión relativamente importante de artefactos en torno al humedal que le da nombre, entre los que se encuentra un gran molino y un raspador de cuarzo, así como cerámicas lisas o con aplicaciones plásticas (cordones, asas). Los carbones hallados en asociación con el elemento durmiente y fragmentos de sendos vasos lisos proporcionaron una fecha bien adentrada en el Bronce inicial (2135-1945 AC) (Fábregas, 1998). Coto da Fenteira, por su parte, es menos expresivo en sus insculturas (6) enmarcables entre los motivos más simples del grupo geométrico, salvo una figura que parece representar un artefacto enmangado de difícil clasificación. El único túmulo documentado es de escasas dimensiones ( $11 \mathrm{~m}$ de diámetro máximo por $0.6 \mathrm{~m}$ de altura) y albergaba una cámara pétrea muy alterada. El repertorio cerámico descubierto en superficie es bastante interesante e incluye un fragmento de campaniforme y otro con decoración de tipo Penha, así como un pezón alargado, un fondo plano y el arranque de un asa igualmente plana. Hay 
que mencionar igualmente el hallazgo de una mano de molino, un pequeño núcleo de sílex y un trozo de arcilla con una impronta vegetal, perteneciente con toda probabilidad al revestimiento de una pared.

La cronología de los restos arqueológicos de Coto da Fenteira sólo puede ser aproximada, entre otras razones por las propias limitaciones que la prospección impone a este respecto. La ubicación temporal del único túmulo es particularmente ambigua, pues construcciones de pequeño tamaño son características tanto de los inicios del megalitismo (final delV milenioAC) como de las manifestaciones funerarias propias ya del Bronce (fin del III primera mitad del II milenio AC), si bien su aparición aislada y lejos de la gran necrópolis sita en la penillanura nos inclinan más por la segunda opción. Tampoco los petroglifos nos permiten mayores precisiones, más allá de una genérica atribución al III milenio o primera parte del II. Las cerámicas por su parte pueden encuadrarse algo mejor: no lejos de Monte Penide la cronología radiométrica nos sitúa con gran precisión el nivel Penha de Lavapés (Cangas de Morrazo) en los siglos XXVI-XXV AC y si bien ignoramos el tipo exacto de campaniforme al que pertenece el fragmento encontrado, su ámbito temporal podría estar con más probabilidad entre los siglos XXV-XX AC; a su vez, los cacharros lisos con fondo plano, asas y decoraciones plásticas podrían tener fechas más tardías, como la ya apuntada para Poza da Lagoa o incluso algo posteriores. El propio muestreo edafológico nos suministra informaciones directas sobre la presencia humana en el área, al documentar un fragmento cerámico liso (de características muy semejantes a los recuperados en prospección) entre los carbones de la base del nivel III (fechados en el segundo cuarto del III milenio AC) y de nuevo en el límite inferior del nivel II, en este caso de minúsculo tamaño (datados hacia el segundo cuarto del II milenio AC).

Cabe preguntarse por la naturaleza de las dispersiones de materiales líticos y cerámicos, situadas a tan corta distancia (menos de $30 \mathrm{~m}$ a veces) de manifestaciones tan llenas de simbolismo como son los grabados rupestres o los túmulos. De nuevo debemos dar una respuesta en términos probabilísticos, pues los indicios disponibles no son concluyentes, pero pensamos que obedecen a la existencia de un asentamiento en las inmediaciones, basándonos en varios criterios: el número de restos cerámicos y el escaso rodamiento de las fracturas, el acabado y grosor de muchas piezas, que apunta a un empleo para el almacenaje o preparación de alimentos, la presencia de material de molienda, en algún caso (Poza da Lagoa) de grandes dimensiones, y el trozo de revestimiento hallado en Fenteira. Finalmente, la preparación de alimentos se ha documentado por la aparición de restos microscópicos de trigo en las superficies activas del durmiente y moviente de molino manual localizados en Poza da Lagoa y Coto da Fenteira, respectivamente (1). En el caso concreto de Coto da Fenteira, creemos que el espacio habitacional (de carácter no completamente sedentario) se encontraría en una leve dorsal que se sitúa al Sur de la cuenca, lo que evitaría la excesiva humedad del terreno topográficamente inferior, permitiría un mejor control visual y resulta coherente con la mayor concentración de hallazgos en la parte de la pista de tierra que discurre en sus inmediaciones.

\section{Reconstrucción paleoambiental: archivos, señales, metodologías}

Los restos materiales de la cultura no son los únicos vestigios de la actividad humana de épocas prehistóricas. La explotación del territorio por parte de los grupos humanos da lugar a la generación de impactos directos e indirectos que dejan su huella en determinados registros, $\mathrm{o}$ archivos paleoambientales, en forma de señales de diversa naturaleza. El ámbito cronológico y la resolución temporal y analítica de la señal son propiedades importantes a tener en cuenta a la hora de abordar las posibilidades de éxito de una reconstrucción que, en todo caso, será siempre parcial y constituirá un palimpsesto de las realidades pretéritas. La integración de todas las señales disponibles y la búsqueda de la coherencia argumental entre ellas constituye, a nuestro juicio, la base de la mejor aproximación posible.

En el noroeste de la Península Ibérica, el estudio de la evolución del paisaje se ha abordado desde muy variadas ópticas y disciplinas, que van desde la Geomorfología, la Edafología, la Arqueología y la Prehistoria, pasando por la Palinología hasta la Geoquímica -esta última de incorporación más reciente-. La información de que disponemos actualmente para el Holoceno muestra una historia dominada por episodios de erosión y sedimentación, cambios en las formaciones superficiales y en

(1) Análisis preliminares realizados por el Dr. Jordi Juan i Tresserras (Universitat de Barcelona), formando parte de un trabajo actualmente en preparación con uno de los autores (R.F.V.). 
las rutas edafogenéticas dominantes a diversas escalas espaciales y temporales, en la cual el peso de los procesos de indución antrópica ha seguido una progresión exponencial. En esta línea de investigación, el estudio de los elementos traza en los suelos ha sido empleado para identificar discontinuidades en los perfiles, en particular aquellas relacionadas con procesos de erosión/sedimentación (E/S) en suelos policíclicos. Entre episodios de E/ $\mathrm{S}$ el suelo tiene una superficie estable, la cual es colonizada por la vegetación y está sujeta a un enriquecimiento en elementos traza por varios mecanismos, tal como han descrito Rose et alii (1979). La meteorización, la edafogénesis, los biociclos (reciclado biológico) y la deposición atmosférica, son los mecanismos de enriquecimiento más importantes. En los suelos ácidos, una elevada proporción de los elementos traza es retenida por los coloides inorgánicos y orgánicos (óxidos de hierro y materia orgánica, principalmente), limitando con ello su movilidad y dando lugar a un aumento de su concentración. En consecuencia, las distintas superficies de los suelos policíclicos, incluyendo las enterradas, presentarán un mayor enriquecimiento en metales traza en relación a otros niveles que nunca hayan ocupado la superficie del terreno. El Ti y el Zr se encuentran entre los elementos más utilizados, por presentar un comportamiento conservador en la edafogénesis, pero otros elementos cuyas concentraciones dependan principalmente de la deposición atmosférica, en sistemas edáficos que favorezcan su preservación, tienen una utilidad potencial para la identificación de las paleosuperficies y de la formación de suelos complejos.

Por ejemplo, Martínez Cortizas et alii (1999a) demostraron que el $\mathrm{Hg}$ cumple estas características en los rankeres atlánticos de Galicia. El ranker atlántico, o suelo humífero coluvial, es un tipo de suelo complejo formado por episodios de adición de material erosionado desde las laderas (Guitián y Carballas, 1968), que suele ocupar posiciones de vaguada o replano -áreas de baja energía- y que contiene, por tanto, señales de la evolución del entorno en el cual se han formado. Así pues, la combinación de estudios geoquímicos y edáficos, junto con la información disponible sobre la evolución prehistórica en áreas específicas, puede ayudar a la reconstrucción de la evolución del paisaje holoceno y a discernir el papel que las actividades humanas tuvieron en dicha evolución.

En este trabajo presentamos el estudio del ranker atlántico de Coto Fenteira (CTF), ubicado en Monte Penide (Pontevedra) y desarrollado a partir de sedimentos graníticos. Se trata de un suelo profundo, con $145 \mathrm{~cm}$ de espesor, de aspecto homogéneo, que ocupa una posición de pie de ladera entre dos oteros. Debido a la aparente homogeneidad del perfil, el muestreo se hizo de forma sistemática, tomando muestras de suelo de $5 \mathrm{~cm}$ de espesor. En las muestras se hicieron las determinaciones analíticas de rutina en edafología y submuestras molidas y homogeneizadas de la fracción tierra fina $(<2 \mathrm{~mm})$ se enviaron al laboratorio EMMA Analytical Inc. de Canadá, donde se determinaron las concentraciones de elementos traza ( Ti, $\mathrm{Cu}, \mathrm{Ga}, \mathrm{Br}, \mathrm{Zr}, \mathrm{Y}, \mathrm{Pb}$ y U) con un analizador multielemental de microsonda de energía dispersada (energy dispersive miniprobe multielement analyzer) (Cheburkin y Shotyk, 1996), técnica que tiene la ventaja de ser no destructiva y poseer límites de detección muy bajos.

También se separó la fracción limo y arcilla $(<50$ $\mu \mathrm{m})$, en la cual se llevó a cabo la determinación del contenido de $\mathrm{Hg}$, usando para ello un equipo LECO-ALTEC AMA-254, que es un analizador espectrofotométrico específico para este elemento. Las medidas se repitieron dos o tres veces por muestra y también se emplearon materiales estándar de referencia (SO-3, Canadá Centre for Mineral and Energy Technology y 1633b, USA National Institute of Standards \& Technology) para la calibración. Finalmente, algunas muestras de suelo (fracción $<50 \mu \mathrm{m}$ ) y carbones recogidos de los perfiles fueron enviados al laboratorio Beta (Miami, EE.UU.), para obtener dataciones radiocarbónicas de los distintos niveles.

\section{ANÁLISIS DEL DEPÓSITO DE COTO DA FENTEIRA}

\section{Morfología y propiedades físico-químicas}

En el ranker atlántico de Coto Fenteira (CTF) hemos identificado cuatro niveles estratigráficos (Fig. 2). En todos se han encontrado carbones, si bien hay profundidades en las cuales la concentración es muy elevada, formando líneas continuas. $\mathrm{La}$ presencia de este rasgo, junto a cambios en las propiedades morfológicas, definen los límites entre los distintos niveles.

El nivel basal (IV) es el de mayor potencia (75 $\mathrm{cm}$ ), su color es negro a negro pardusco hacia techo y marrón amarillento a amarillo oscuro en pro- 

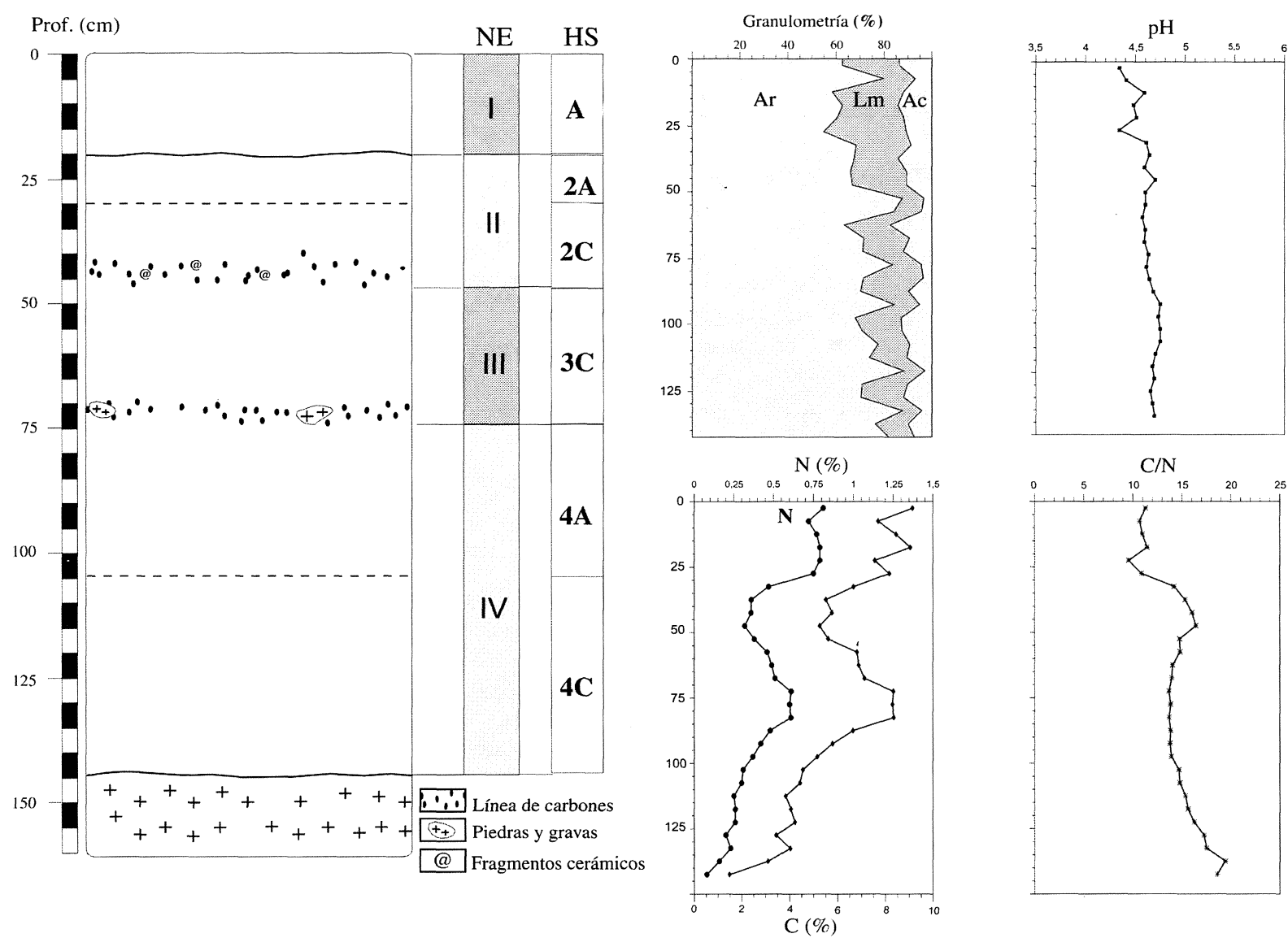

Fig. 2. Niveles estratigráficos (NE), ciclos y horizontes de suelo (HS), rasgos más notables y propiedades físico-químicas del ranker atlántico de Coto da Fenteira (Monte Penide, Redondela, Pontevedra). Granulometría: Ar, arenas; Lm, limos; Ac, arcillas.

fundidad. El límite con el nivel III está marcado por una una discontinuidad clara, expresada por la presencia de una línea de piedras de granito y una concentración de carbones a $70 \mathrm{~cm}$ de profundidad. A esa cota, también se ha encontrado un fragmento cerámico (de $40 \times 28 \times 9 \mathrm{~mm}$ ). El nivel III, de $25 \mathrm{~cm}$ de espesor, es algo más heterogéneo, de color pardo rojizo en la base y negro en la parte superior.

El nivel II tiene también unos $25 \mathrm{~cm}$ de espesor y es de color negro pardusco a techo y negro pardusco a pardo amarillento oscuro en la base. La presencia de abundantísimos carbones a una profundidad de 40-45 cm, determina su límite inferior. En esta línea de carbones se han encontrado pequeños fragmentos cerámicos, cuyo eje mayor no supera los 5 $\mathrm{mm}$. El nivel I es el de menor potencia, $20 \mathrm{~cm}$, y es de color marrón grisáceo oscuro a techo y negro en profundidad.

Desde el punto de vista edáfico, se trata en con- junto de un suelo ácido (pH entre 4.4 y 4.8), de elevada porosidad y buena conductividad hidráulica, textura entre franco-arenosa y arenoso-franca, rico en materia orgánica (contenido mínimo de un 2'5\% y máximo de un $15 \%$ ) y pobre en fósforo y otros nutrientes esenciales. Los niveles estratigráficos, definidos basándonos en las propiedades morfológicas, coinciden con diferentes ciclos de suelo.Así lo reflejan los perfiles verticales de propiedades físico-químicas como el contenido de carbono (C) y nitrógeno $(\mathrm{N})$-que muestran máximos subsuperficiales- o de las fracciones granulométricas (Fig. 2). Cabe destacar, no obstante, que la relación carbono/nitrógeno $(\mathrm{C} / \mathrm{N})$ presenta un cambio notable en los ciclos I y II, con valores de 9-10, frente a los ciclos basales, en los cuales tiende a ser superior a 14 , en particular en la base del ciclo II y en los niveles más profundos del suelo. Los valores medios para los suelos desarrollados sobre granitos en Galicia, con independencia de su uso, se encuentran 
entre 12.5 y 14.9 (2). Esta relación es utilizada habitualmente como un indicador del grado de evolución y origen de la materia orgánica del suelo, por lo que los bajos valores obtenidos en los ciclos superiores parecen indicar un cambio importante en la composición de las comunidades vegetales del área de Coto Fenteira, en el intervalo temporal representado por los dos últimos ciclos.

Además de la superficie actual del ranker, tanto los datos morfológicos como los físico-químicos nos han permitido identificar, al menos, otros dos niveles con propiedades de horizontes A úmbricos (mayor contenido de materia orgánica, muy baja saturación de bases, elevada saturación por aluminio en el complejo de intercambio catiónico, menor densidad del suelo; etc...), situados entre $20-35 \mathrm{~cm}$ y entre $80-105 \mathrm{~cm}$ de profundidad respectivamente. En conjunto, estos resultados sugieren la existencia de superficies enterradas y por tanto, que el perfil es policíclico y contiene paleosuelos de épocas pretéritas.

\section{Elementos traza: geoindicadores de superficies enterradas}

Tal como se ha mencionado en la introducción, los modelos geoquímicos de concentración de elementos traza en el suelo señalan que hay diversos procesos que hacen que éstos tiendan a concentrarse en su superficie (el epipedon u horizonte A). Asumiendo estos modelos, la existencia de niveles subsuperficiales enriquecidos en elementos traza, unido a los rasgos estratigráficos y las propiedades edáficas ya descritas, serían un argumento de peso para la identificación de las paleosuperficies.

En la tabla I se han resumido los datos referentes a los elementos analizados en $\mathrm{CTF}(\mathrm{Ti}, \mathrm{Cu}, \mathrm{Ga}$, $\mathrm{Br}, \mathrm{Y}, \mathrm{Zr}, \mathrm{Hg}, \mathrm{Pb}$ y U). Dado que las tendencias de la acumulación en los perfiles verticales pueden estar sujetas a variaciones significativas, debidas a cambios en la composición mineralógica u otras propiedades del suelo, resulta más adecuado emplear los factores de enriquecimiento (FE). Estos se obtienen normalizando las concentraciones a las de un elemento conservador, es decir, cuya fuente principal sea la meteorización de la corteza terrestre (Schütz y Rahn, 1982), y que además no sea móvil en el sistema edáfico que se analiza. Entre los ele-

(2) A. Riveiro (1992): Datos para la evaluación agronómica de los suelos de la provincia de La Coruña. Tesis de Doctorado, Facultade de Bioloxía. Santiago de Compostela.

\begin{tabular}{lccccccccc}
\hline & $\mathbf{T i}$ & $\mathbf{C u}$ & $\mathbf{G a}$ & $\mathbf{B r}$ & $\mathbf{Y}$ & $\mathbf{Z r}$ & $\mathbf{H g}$ & $\mathbf{P b}$ & $\mathbf{U}$ \\
\hline Conc. & 908.0 & 7.0 & 17.7 & 230.2 & 31.4 & 118.6 & 101.2 & 24.5 & 51.3 \\
Max. & 1141 & 11.7 & 21.2 & 335.1 & 40.0 & 185.0 & 245.3 & 34.0 & 85.2 \\
Mín. & 701 & 2.1 & 14.5 & 91.6 & 20.5 & 93.0 & 53.1 & 21.0 & 17.8 \\
D. Est. & 119.3 & 2.3 & 1.5 & 53.5 & 4.5 & 19.0 & 35.0 & 3.8 & 20.3 \\
CV-C & 13.1 & 32.8 & 8.5 & 23.2 & 14.3 & 16.0 & 34.6 & 15.5 & 39.6 \\
FE & --- & 4.46 & 1.32 & 3.26 & 1.98 & 1.15 & 1.44 & 1.56 & 1.98 \\
Max. & --- & 8.64 & 1.61 & 5.58 & 3.03 & 1.65 & 4.25 & 2.38 & 4.05 \\
Mín. & --- & 1.02 & 0.96 & 1.00 & 1.0 & 0.90 & 0.78 & 1.02 & 0.63 \\
D. Est. & --- & 1.89 & 0.15 & 0.98 & 0.51 & 0.18 & 0.68 & 0.39 & 0.99 \\
CV-FE & & 42.4 & 11.4 & 30.1 & 25.7 & 15.6 & 47.2 & 25.0 & 50.0 \\
\hline
\end{tabular}

Tab. 1. Valores medios, máximo, mínimo, desviaciónestandar (D.Est.) y coeficientes de variación (CV-C y CV-FE) para las concentraciones (Conc) y los factores de enriquecimiento (FE) de los elementos traza analizados en Coto da Fenteira (Monte Penide, Redondela, Pontevedra) (todas las concentraciones expresadas en ppm, a excepción de las de $\mathrm{Hg}$ que están en $\mathrm{ppb}$ ).

mentos más utilizados con este fin se encuentran el Ti, el Sc y el Al (Shotyk, 1996). Los FE así calculados sirven como indicadores de la intensidad de la acumulación por encima de los valores de fondo, que dependen de la meteorización de la roca.

Así pues, en la tabla I, además de las concentraciones se dan los FE para cada elemento, calculados normalizando la relación elemento/Ti a la obtenida en la muestra mas profunda del perfil -considerando que ésta es representativa del material de partida del suelo-. Los elementos que hemos analizado muestran dos tipos de comportamiento geoquímico: uno conservador y otro no conservador. Al primer grupo pertenecen el Ti, el $\mathrm{Zr}$ y el Ga; mientras que al segundo pertenecen $\mathrm{Cu}, \mathrm{Br}, \mathrm{Y}, \mathrm{Hg}$, $\mathrm{Pb}$ y $\mathrm{U}$. En la figura 3 pueden verse ejemplos de estos tipos de comportamiento. Los perfiles verticales de $\mathrm{Ga}$ y $\mathrm{Zr}$ tienen muy pequeñas variaciones en los FE (coeficientes de variación de 11.4 y 15.6), siendo el valor medio de 1.32 y 1.15 , repectivamente. Esto indica que el material de partida de CTF es homogéneo en cuanto a su origen litológico, y que el área fuente de materiales para el suelo es restringida. Esto último es apoyado también por el análisis geomorfológico del área de captación de Coto da Fenteira.

Por lo que respecta a los elementos no conservadores, sus concentraciones y factores de enriquecimiento tienen coeficientes de variación elevados y presentan máximos que no coinciden con la superficie actual del suelo. Entre ellos $\mathrm{Cu}, \mathrm{Y}, \mathrm{Pb}$ y $\mathrm{U}$ están presentes en el material geológico (los granitos) en concentraciones del orden de las determinadas en el suelo: $1.5-2.5 \mathrm{ppm}$ de $\mathrm{Cu}, 10-20 \mathrm{ppm}$ deY, 2025 ppm de $\mathrm{Pb}$ y $10-15 \mathrm{ppm}$ de $\mathrm{U}$; mientras que las concentraciones de $\mathrm{Br}$ y $\mathrm{Hg}$ en la roca, son extraordinariamente bajas en relación a las del suelo: 1-2 

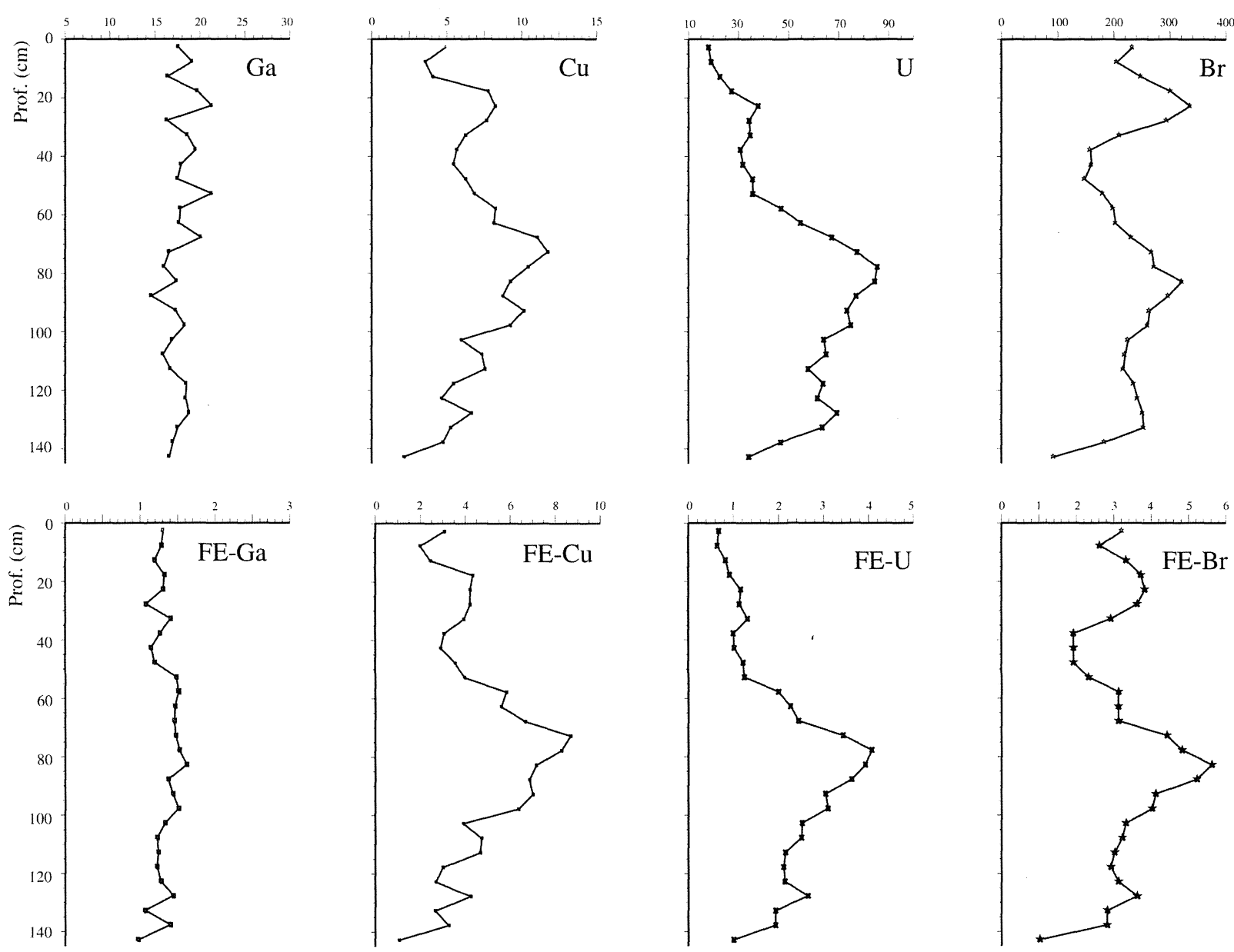

Fig. 3. Ejemplos de variaciones verticales en la concentración y los factores de enriquecimiento (FE) de algunos de los elementos traza medidos en el ranker atlántico de Coto da Fenteira (Monte Penide, Redondela, Pontevedra). Todas las concentraciones en ppm a excepción de las de Hg que están en ppb.

ppm de Br y 1-2 ppb de Hg. Esto quiere decir, respecto a su comportamiento geoquímico, que los mecanismos de enriquecimiento de los primeros están relacionados esencialmente con los procesos edafogenéticos y/o el reciclado biológico, mientras que las concentraciones de los segundos lo están, casi exclusivamente, con la deposición atmosférica sobre la superficie del terreno; siendo la fuente de $\mathrm{Br}$ los aerosoles marinos y en el caso del $\mathrm{Hg}$ el vulcanismo y la degasificación de la corteza terrestre y los océanos (Nriagu, 1979). Por tanto, estos dos últimos elementos han de considerarse como los marcadores más fiables de la posición de las paleosuperficies enterradas, muy en particular el $\mathrm{Hg}$ dada su baja o nula movilidad en estos ambientes edáficos.

Los FE medios indican que el $\mathrm{Cu}$ muestra una concentración relativa que es de casi 4.5 veces la del material de partida, de unas 3.3 veces para $\mathrm{Br}$, de casi 2 veces para elY y el U, y del orden de 1.5 veces para el $\mathrm{Hg}$ y el $\mathrm{Pb}$. No obstante, en todos los casos los FE más elevados (8.6, 5.6, 3.0, 4.3, 2.4, y 4.1, para $\mathrm{Cu}, \mathrm{Br}, \mathrm{Y}, \mathrm{Hg}, \mathrm{Pb}$ y U, respectivamente) se encuentran entre $75-85 \mathrm{~cm}$ de profundidad. $\mathrm{El} \mathrm{Br}$ y el $\mathrm{Hg}$ tienen el máximo a $80-85 \mathrm{~cm}$, mientras que los demás lo tienen a 75-80, o el valor para esta última muestra es muy próximo al de la subyacente. Por tanto, estos indicadores geoquímicos sugieren que existió una superficie estable del suelo que ahora se encuentra a $80-85 \mathrm{~cm}$ de profundidad en el perfil (PLsp-I), y que fue enterrada por sedimentos procedentes de las laderas. Dado que la erosión comienza por removilizar los horizontes superficiales, los primeros materiales en llegar ya vendrían enriquecidos en elementos traza, en particular en aquellos cuyos mecanismos dominantes son la edafogé- 
nesis y el reciclado biológico. De ahí que los elementos de este grupo muestren FE similares a los de la paleosuperficie en las muestras que se encuentran justo por encima de la misma. Conforme la erosión progresa y se exponen en las laderas horizontes más profundos de los suelos, los sedimentos que llegan al ranker comienzan a mostrar concentraciones inferiores.

Además de esta paleosuperficie, los factores de enriquecimiento de $\mathrm{Cu}, \mathrm{Br}$ y $\mathrm{Hg}$ muestran un máximo secundario a $20-30 \mathrm{~cm}$ de profundidad, indicando la presencia de una segunda superficie enterrada (PLsp-II).

Por tanto, al igual que ocurría con los rasgos morfológicos y estratigráficos y las propiedades físico-químicas, el análisis del enriquecimiento en elementos traza apoya la interpretación de que el ranker de Coto da Fenteira contiene dos superficies enterradas (PLsp-I y PLsp-II), que fueron estables durante un periodo de tiempo más o menos prolongado. Estos análisis también ponen de manifiesto, que no hay evidencias de etapas de estabilidad distintas de las representadas por la superficie actual y estas dos superficies enterradas.

\section{Génesis del suelo y cronología de los episodios}

La formación del ranker de Coto da Fenteira ha sido pues el resultado de un balance entre fases de estabilidad en el paisaje, con predominio de las rutas progresivas de evolución de suelos, y fases de inestabilidad, con predominio de las rutas regresivas (ambas en el sentido de Johnson y Watson-Stegner, 1987). Las primeras favorecerían la diferenciación del perfil, con un aumento de la anisotropía morfológica, físico-química y mineralógica; mientras que las segundas habrían dado lugar a una pérdida o ralentización de la diferenciación, promovida por factores tendentes a la isotropía. A juzgar por los datos obtenidos, el suelo que ocupaba la paleosuperficie más antigua que se conserva en CTF, PLspI, se vio sometido a un cambio drástico en su tendencia evolutiva, desencadenado por procesos que operaron a nivel de paisaje sobre los suelos de ladera, dando lugar en éstos a una erosión progresivamente más intensa y a la concomitante sedimentación sobre la paleosuperficie de CTF. La intensidad de esta erosión pronto superó un límite crítico, de tal forma que la acreción (aumento del espesor del suelo por adiciones en su superficie) se volvió noasimilativa; es decir, que los procesos edafogenéti- cos no fueron capaces de producir la transformación del material incorporado. Cabe mencionar, no obstante, que dicho material, al proceder de suelos de ladera, ya presenta un grado de evolución pedogenética, aunque éste no fue adquirido in situ en relacióna la posición del paleosuelo, por lo que es considerado como un sedimento o un material-suelo de partida. En este sentido, debemos entender que son los procesos de ladera los responsables de la formación del ranker o, si se quiere, de la rankerización del suelo.

En la tabla II se recogen las dataciones radiocarbónicas realizadas en Coto da Fenteira. Basándonos en ellas, podemos fijar el inicio de la edafogénesis del paleosuelo de la superficie PLsp-I a comienzos del Holoceno (7060-6625 AC) (Fig. 4). La estabilidad habría durado hasta la fase de óptimo (46054340 AC), reflejándose en la acumulación de materia orgánica (melanización) y elementos traza. La líneạ de carbones del nivel III, datada hacia el 2900$2590 \mathrm{AC}$, probablemente relacionada con una quema de vegetación de origen antrópico, marca el comienzo de una crisis erosiva de elevada intensidad, responsable de la sedimentación de materiales de ladera sobre el paleosuelo. La datación hecha sobre materia orgánica en el mismo nivel pero justo por encima de la línea de carbones, de 2680-2310 $\mathrm{AC}$, se solapa ligeramente o es inmediatamente posterior a la de los carbones; mientras que la parte superior del nivel III, de edad 1955-1620AC (sobre materia orgánica), y la línea de carbones de la base del nivel II (segunda quema), con edad 1880$1420 \mathrm{AC}$, se solapan casi íntegramente. Esto indica que no sólo los procesos erosivos son coetáneos con las quemas sino que también es muy probable que se hayan reiterado en el tiempo. Si se tiene en

\begin{tabular}{|c|l|c|c|c|}
\hline $\begin{array}{c}\text { Profundidad } \\
(\mathrm{cm})\end{array}$ & \multicolumn{1}{|c|}{ MATERIAL } & $\begin{array}{c}\text { EDAD } \\
\text { CONVENCIONAL }\end{array}$ & $\begin{array}{c}\text { EDAD } \\
\text { CALIBRADA }\end{array}$ & Código \\
\hline $20-25$ & Materia orgánica & $2120 \pm 60 \mathrm{BP}$ & $365 \mathrm{BC}-5 \mathrm{AD}$ & $\beta-130235$ \\
\hline $40-45$ & Carbones & $3330 \pm 100 \mathrm{BP}$ & $1880-1420 \mathrm{BC}$ & b-124913 \\
\hline $45-50$ & Materia orgánica & $3470 \pm 70 \mathrm{BP}$ & $1955-1620 \mathrm{BC}$ & b-130236 \\
\hline $60-65$ & Materia orgánica & $4000 \pm 70 \mathrm{BP}$ & $2680-2310 \mathrm{BC}$ & b-130237 \\
\hline $70-75$ & Carbones & $4160 \pm 50 \mathrm{BP}$ & $2900-2590 \mathrm{BC}$ & b-124914 \\
\hline $80-85$ & Materia orgánica & $5630 \pm 70 \mathrm{BP}$ & $4605-4340 \mathrm{BC}$ & b-130238 \\
\hline $135-140$ & Materia orgánica & $7930 \pm 70 \mathrm{BP}$ & $7060: 6625 \mathrm{BC}$ & b-130240 \\
\hline
\end{tabular}

Tab. 2. Dataciones radiocarbónicas realizadas en el ranker atlántico de Coto da Fenteira (Monte Penide, Redondela, Pontevedra). Se indica el material sobre el que se llevó a cabo la datación, la edad convencional y la edad calibrada.

T. P., 57, n. $^{\circ} 1,2000$ 


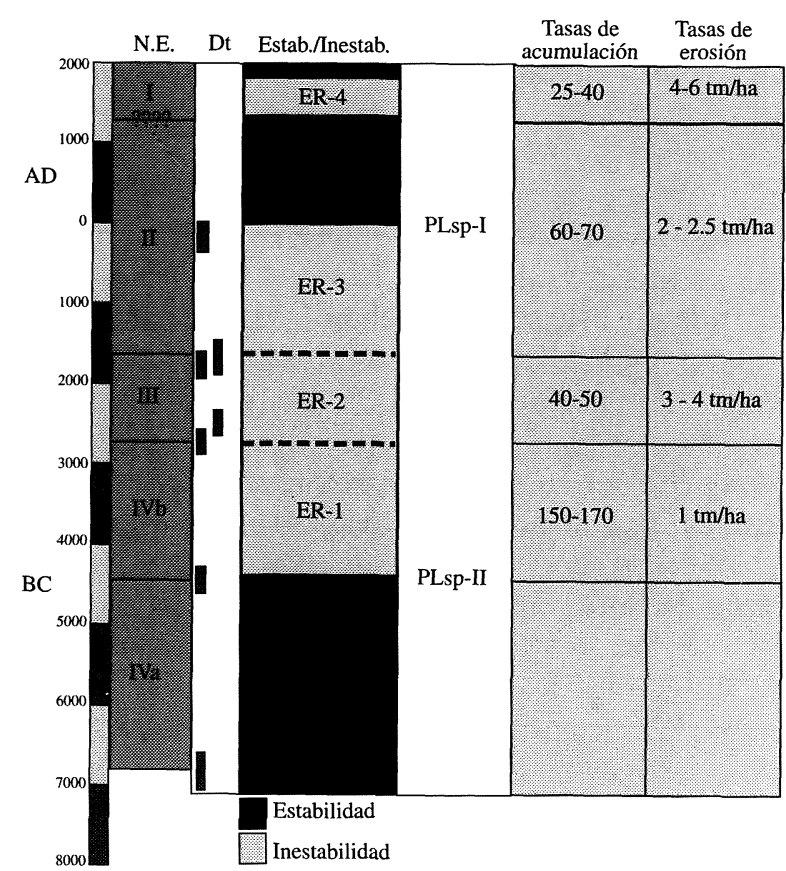

Fig. 4. Síntesis cronológica de la génesis y los procesos que aparecen reflejados en el ranker atlántico de Coto da Fenteira (Monte Penide, Redondela, Pontevedra). NE: niveles estratigráficos; Dt: dataciones, en sombreado las realizadas sobre carbones, el resto sobre materia orgánica del suelo; ER-1 a ER-4: principales episodios erosivos; PLspII y PLsp-I, paleosuperficies del ranker; las tasas de acumulación están en años necesarios para producir la acumulación de $1 \mathrm{~cm}$; tasas de erosión equivalentes, expresadas en toneladas métricas por hectárea y año.

cuenta la edad de la línea de carbones que señala el inicio del enterramiento de la superficie PLsp-II, de 2900-2590AC, la inestabilidad del paisaje se habría mantenido entre esta fecha y el $365 \mathrm{AC}-5 \mathrm{AD}$ : un período de inestabilidad sostenida de unos 2300 a 3000 años.

Debemos señalar, por otro lado, que previa a la sedimentación causada por la quema más antigua tuvo lugar otra, fruto de un episodio erosivo anterior, tal como señalan los FE de algunos elementos $(\mathrm{Cu}, \mathrm{Y}, \mathrm{Pb}$ y U). Así pues, entre el 4605-4340 AC y el 2900-2590 AC podría haberse producido ya una cierta presión sobre los suelos de ladera, que desencadenaría una erosión de baja intensidad que dio lugar a una acreción superficial asimilativa -nivel identificado en la figura 4 como IVb-. Este podría no haber sido un proceso local, ya que unos kilómetros al sur de Cota da Fenteira, en Mougas, Costa et alii (1996) dataron un nivel de carbones en un suelo policíclico, marcador de un proceso erosivo/ sedimentario, en el $5530 \pm 60 \mathrm{BP}$ que en cronología calibrada da un 4510-4310AC, plenamente coincidente con Coto da Fenteira.

Finalmente, la superficie PLsp-II también es enterrada durante un episodio que es posterior al $365 \mathrm{AC}-5 \mathrm{AD}$. No disponemos de dataciones radiocarbónicas para fijar una cronología mas o menos precisa para este evento. No obstante, podemos obtener una estimación por comparación con PLsp$\mathrm{I}$, asumiendo que el enriquecimiento en elementos traza es proporcional al tiempo de estabilidad de la superficie. Según las dataciones disponibles, PLspI habría sido estable del orden de unos 2.400 años, tiempo durante el cual los elementos traza se habrían ido acumulando. Estableciendo una proporcionalidad entre los FE de ambas paleosuperficies, las estimaciones del tiempo de estabilidad para PLsp-II son del orden de 1300 a 1600 años, es decir, que la crisis erosiva habría tenido lugar entre hace unos 800 y unos 500 años.

Por otro lado y como ya hemos mencionado, la relación $\mathrm{C} / \mathrm{N}$ de los niveles II y I apoya un posible cambio en la vegetación del área (tal vez de bosque o matorral a pradera o cultivo), que ahora podríamos acotar a partir del 1880-1420 AC. Cabe decir, a este repecto, que dicho cambio habría afectado a los suelos de las laderas y no al área del ranker de Coto da Fenteira. Obviamente, el material que es sedimentado procede de allí, pero además no hay en las propiedades de este suelo evidencias de una modificación antrópica directa posterior a la sedimentación (los niveles de P, por ejemplo, son muy bajos).

No obstante, la evolución en Coto da Fenteira ha estado, cuando menos, inducida por las actividades humanas en el entorno. Así lo atestiguan las concentraciones de carbones y la presencia de fragmentos cerámicos en el perfil, además de otros datos arqueológicos que se discuten más adelante. Esta actividad se tradujo en la erosión de los suelos de ladera, debida a la deforestación por quema, y en un crecimiento del espesor del suelo por sedimentación que desencadenó la rankerización. De lo expuesto hasta aquí, se deduce la existencia de cuatro episodios de erosión: ER-1, responsable de la acreción asimilativa en PLsp-I, nivel IVb; ER-2, responsable de la formación del nivel III; ER-3, que da lugar a la formación del nivel II; y ER-4, que da lugar al nivel I. Teniendo en cuenta la profundidad de cada uno de los niveles de acreción y las edades radiocarbónicas calibradas obtenidas (con su desviación $2 \mathrm{~s}$ ), es posible obtener una aproximación a la intensidad erosivo/sedimentaria de cada fase. La 
primera fase de acreción del ranker habría resultado en la formación de $1 \mathrm{~cm}$ de suelo cada 150-170 años $(10 \mathrm{~cm}$ acumulados entre el 4605-4340 BC y 2900-2590 BC); la segunda tiene una tasa de sedimentación de $1 \mathrm{~cm}$ cada 40-50 años; la formación del nivel II ocurriría con una tasa de $1 \mathrm{~cm}$ cada 6070 años; mientras que la tasa de sedimentación para el nivel I es de $1 \mathrm{~cm}$ cada 25-40 años (estimada a partir de la edad calculada empleando los factores de enriquecimiento). Las tasas de erosión equivalentes -considerando que la densidad de estos suelos se encuentra entre 0.8 y $1.2 \mathrm{Mg} \mathrm{m}^{-3}$, con una media de $1.0 \mathrm{Mg} \mathrm{m}^{-3}$ - serían de $1 \mathrm{tm}$ por hectárea y año para ER-1, de 3-4 tm por hectárea y año para ER-2, de 2-2.5 tm por hectárea y año para ER-3 y de 4-6 tm por hectárea y año para ER-1. Debe entenderse que se trata de estimaciones mínimas para las tasas de erosión, pues en su cálculo se presupone que todo el material erosionado habría alcanzado el área de Coto da Fenteira y no habría sido removilizado ulteriormente, algo poco probable. Aún así, ER-2 se manifiesta como una perturbación profunda y sin precedente en la evolución del paisaje, ya que la tasa de erosión es de tres a cuatro veces superior a la del episodio previo; lo cual induce a pensar en un cambio sustancial en las relaciones hombre/medio en Monte Penide, a partir del 2900-2590 AC. Esta dinámica no se cortaría de forma clara hasta una época muy posterior pues, aunque las tasas de erosión disminuyen ligeramente, no es hasta el $365 \mathrm{AC}-5 \mathrm{AD}$ en que aparece reflejado un episodio de estabilidad en el ranker.

No obstante, a la hora de valorar el efecto de la actividad humana en la evolución del paisaje, se debe tener en cuenta que otros cambios ambientales, como los climáticos, podrían haber jugado un papel importante. El registro más fiable de los paleoclimas del Holoceno final en el Noroeste de la Península Ibérica (Martínez Cortizas et alii, 1999b) indica que, los procesos erosivos ER-2 y ER-3, aquí descritos, son coetáneos con una fuerte regresión climática que finalizó hacia el $3000 \mathrm{BP}$ (período de la Neoglaciación). De igual modo, la edad estimada para el episodio ER-4 se solaparía al menos en parte con el desarrollo del último periodo frío relevante, la Pequeña Edad del Hielo. Mientras que las edades de las superficies PLsp-I y PLsp-II las remiten a sendos periodos de bonanza climática: el óptimo holoceno y el periodo cálido que comienza poco antes del cambio de era y finaliza hacia el 1500 BP. Estos resultados, en consonancia con las investigaciones geoarqueológicas y paleoambientales realizadas hasta el momento (Martínez Cortizas y Moares Domínguez, 1995; Martínez Cortizas, 1996) sugieren que, en buena medida, la actividad humana ha estado en metacronicidad con los cambios de origen natural. El desarrollo de las actividades se ha acoplado a las condiciones ambientales y la intensidad de los procesos de indución antrópica ha dependido, a su vez, de la sensibilidad del medio en cada momento de su estado evolutivo; lo que hemos dado en denominar carga crítica del medio (Martínez Cortizas y Llana, 1996). Quiere esto decir, que en periodos de degradación climática la capacidad del medio para amortiguar los efectos de las actividades humanas es menor y los procesos de cambio inducido se ven intensificados.

\section{CONCLUSIONES}

Los resultados que acabamos de exponer permiten una lectura metodológica y al mismo tiempo proporcionan argumentos para terciar en un debate que tiene que ver con la naturaleza de las actividades de los grupos humanos del Noroeste de la Península Ibérica durante el III/II milenios, así como el ritmo y la continuidad del impacto de aquellas sobre el entorno. Comenzando por el primer aspecto, el método de análisis de suelos expuesto aquí abre nuevas vías para el conocimiento de la evolución del medio que pueden completar o, en su caso, cubrir la falta de otras fuentes de información. Los estudios edafológicos y geoquímicos efectuados nos revelan la dilatada cronología y compleja génesis de un depósito aparentemente homogéneo y deben servir de paso para poner un punto de prudencia, tanto en las apreciaciones estratigráficas hechas por el arqueólogo meramente de visu como en el actualismo subyacente a muchas investigaciones sobre la relación de las sociedades humanas con su entorno en el curso del Holoceno.

Por lo que respecta a la diacronía de las actividades humanas, tal como se reflejan en el depósito de Coto da Fenteira, tenemos un primer punto de interés en la parte superior del nivel IV donde hacia el 4600-4300AC se constata un primer, y leve, episodio erosivo que podemos atribuir con probabilidad a las primeras actividades agropastoriles, con su cortejo de quemas y la consiguiente exposición del suelo en las áreas de pendiente. Es interesante anotar la genérica coincidencia temporal con indicadores semejantes en depósitos cercanos como el de Mougás (Costa et alii, 1996) o en los registros 
polínicos (Ramil, 1993), así como la precedencia en unos pocos siglos de estas prácticas productoras con respecto a la aparición del primer megalitismo (4300-4200 AC). No es casual el hecho de que Monte Penide se enclave en el Suroeste de Galicia, una zona donde se conocen una serie de yacimientos al aire libre o bajo abrigo con cerámicas inciso/ impresas, que se atribuyen al Neolítico inicial regional (Fábregas y Suárez, 1999).

El segundo episodio erosivo, que da lugar a la formación del nivel III a partir de los inicios del III milenio AC, coincide con una fase de auge de los grupos humanos entre el final del Neolítico y los comienzos de la Edad del Bronce, caracterizada por la multiplicación de asentamientos, que además aumentan de tamaño y disponen de una cultura material más rica y diversificada (Fábregas y RuizGálvez, 1997), de lo que son muestra las cerámicas inciso-metopadas de tipo Penha y, posteriormente, la alfarería campaniforme. Todos estos cambios en el registro arqueológico se fundamentan en el proceso de consolidación del sistema agropastoril, observable en los análisis polínicos y carpológicos, o-mucho más escasos por problemas postdeposicionales-paleontológicos (Fábregas et alii, 1997). Lógicamente, el impacto de esas actividades humanas se manifiesta en un incremento correlativo de los procesos erosivos en áreas de ladera, que quedan expuestas a los arrastres motivados por la disminución de la cobertera vegetal a consecuencia de los incendios, un fenómeno que se verá coadyuvado por el enfriamiento climático que tiene lugar a lo largo del III milenio AC y, más atenuado-, durante parte del II (Martínez Cortizas et alii, 1999b).

Otro episodio erosivo, si bien algo menos violento que el precedente, tiene lugar a partir de los siglos XX/XIX AC y no finaliza hasta bien entrado el I milenioAC. Coincide parcialmente con un período, comenzando a finales del Bronce inicial y prolongado hasta la transición Bronce/Hierro, tildado de "edad oscura" a causa de la alegada ausencia de restos habitacionales o funerarios (Peña y Rey, 1993). En otro lugar (Fábregas, 1995; Fábregas y Bradley, 1995) hemos expuesto nuestro desacuerdo con la idea de una regresión socioeconómica durante el II milenio, argumentando que más bien habría tenido lugar una "evolución silenciosa", patente en la continuación de la deforestación y en las innovaciones tecnológicas y formales en el campo de la metalurgia. La "invisibilidad arqueológica" de este proceso se debería fundamentalmente a los cambios que se registran en las costumbres funerarias, así como a la documentada tendencia a la fragmentación del hábitat, adaptándose a la constitución de unidades sociales de menor tamaño. A este proceso de desagregación -que no disolución- social podría haber contribuido un mayor énfasis en las actividades ganaderas, sin que ello implique un abandono de la agricultura (que tenemos documentada indirectamente en los molinos manuales analizados, así como en los restos de vasos de almacenaje). La mayor importancia de las prácticas pastoriles estaría indicada por la tendencia a situar los asentamientos de esta época (y esto se repite por todo Monte Penide) en las inmediaciones de vaguadas o brañas, que actúan como reservas de pasto fresco, especialmente idóneas para el ganado bovino (Méndez, 1998). La continuidad de la presión humana sobre el medio en las inmediaciones de Coto da Fenteira durante todo el II milenio apoya nuestra tesis de una "evolución silenciosa", y la caída detectada en las relaciones carbono/nitrógeno $(\mathrm{C} / \mathrm{N})$ en el nivel II apuntan en la misma dirección: la persistencia durante esa etapa de actividades de clareo en las laderas que dominan Coto da Fenteira desde el Sur, impidiendo la regeneración del bosque. Creemos más lógico, por otra parte, que esa perseverante roza obedecería más a la necesidad de favorecer la extensión y mantenimiento de los pastizales que a la práctica de la agricultura, especialmente complicada a causa de las fuertes pendientes.

\section{BIBLIOGRAFÍA}

Cheburkin, A.K. y Shotyk, W. (1996): “An Energy-dispersive Miniprobe Multielement Analyzer (EMMA) for direct measurement of $\mathrm{Pb}$ and other trace elements in peats". Fresenius Journal of Analytical Chemistry, 354: 688-691.

Costa, M.; Martínez Cortizas, A. y Pérez Alberti, A. (1996): "Caracterización físico-química do depósito litoral de Mougás (Pontevedra): implicacións morfoxenéticas". En A. Pérez Alberti, P. Martini, W. Chesworth y A. Martínez Cortizas (eds.): Dinámica y Evolución de Medios Cuaternarios. Xunta de Galicia. Santiago de Compostela: 431-440.

FÁbREGAS VALCARCE, R. (1995): "La realidad funeraria en el Noroeste del Neolítico a la Edad del Bronce". En R. Fábregas, F. Pérez y C. Fernández (eds.): Arqueoloxía da Morte. Ayuntamiento de Xinzo de Limia. Xinzo de Limia: 95-125.

- (1998): "Petroglifos y asentamientos: el caso de Monte Penide (Redondela, Pontevedra)". Boletín del Seminario de Arte y Arqueología de Valladolid, 64: 91-116. 
FÁbregas Valcarce, R. y Bradley, R. (1995): “El silencio de las fuentes: prácticas funerarias en la Edad del Bronce del Noroeste y su contexto europeo". Complutum, 6: 153-166.

FÁbregas Valcarce, R. y Ruiz-Gálvez, M. (1997): “El Noroeste de la Península en el III y II $^{\circ}$ Milenios. Propuestas para una síntesis“. Homenaje a la prof. M. Gil Mascarell, 2, Saguntum, 30: 191-216.

FÁbregas Valcarce, R. y SuÁrez Otero, J. (1999): “El proceso de neolitización de Galicia". Actas del II Congrés del Neolític a la Península Ibèrica. Valencia. Saguntum Extra, 2: 541-548.

Fábregas Valcarce, R.; Fernández Rodríguez, C. y RAmil Rego, P. (1997): "La adopción de la economía productora en el Noroeste peninsular". Actas del Coloquio Internacional $O$ Neolítico Atlántico $e$ as Orixes do Megalitismo. Santiago de Compostela: 463484.

Guitián, F. y Carballas, T. (1968): "Suelos de la zona húmeda española. III. El ranker atlántico". Anales de Edafología y Agrobiología, 27: 57-73.

JOHNSON, D.L. y WATSON-STEGNER, D. (1987): "Evolution model of pedogenesis". Soil Science, 143: 349-366.

Martínez Cortizas, A. (1996): "Evolución de los suelos durante el Cuaternario reciente en las sierras septentrionales de Galicia (NW de España)". EnA. PérezAlberti y A. Martínez Cortizas (coords.): Avances en la Reconstrucción de las Áreas de Montaña Lucenses. Monografías GEP 1. Diputación Provincial de Lugo, Lugo: 137-161.

Martínez Cortizas, A. y Llana Rodríguez, C. (1996): Una aproximación intedisciplinar a la contextualización de los yacimientos al aire libre de Galicia". Almadan, 5: 23-26.

Martínez Cortizas, A. y Moares Domínguez, C. (1995): Edafología y Arqueología. Estudio de yacimientos arqueológicos al aire libre en Galicia. Xunta de Galicia,. Santiago de Compostela.

Martínez Cortizas A.; Looijaard, A.; Franco Maside, S. y GARCíA-Rodeja, E. (1999a): "Complex soil evolution and trace metals: the case of $\mathrm{Hg}$ in the atlantic ranker". Proceedings of the $5^{\text {th }}$ International Conference on the Biogeochemistry of Trace Elements, I.Viena: 106-107.

Martínez Cortizas, A.; Pontevedra Pombal, X.; Nóvoa MuÑoz, J.C.; GarCía-Rodeja, E. y ShotyK. W. (1999b): "Mercury in a Spanish peat bog: archive of climate change and atmospheric metal deposition". Science, 284: 939-942.

Méndez FernÁndeZ, F. (1998): "Definición y análisis de poblados de la Edad del Bronce en Galicia“. En R. Fábregas (ed.): A Idade do Bronce en Galicia: novas perspectivas. Cadernos do Seminario de Sargadelos, 77. A Coruña: 153-189.

NRIAGU, J.O. (1979): "Global inventory of natural and anthropogenic emissions of trace elements to the atmosphere". Nature, 279: 409-411.

Peña Santos, A. y Rey García, J. (1993): "El espacio de la representación. El arte rupestre galaico desde una perspectiva territorial“. Revista de Estudios Provinciais, 10: 11-50.

RAMIL REGO, P. (1993): "Evolución climática e historia de la vegetación durante el Pleistoceno Superior y el Holoceno en las regiones montañosas del Noroeste ibérico". En A. Pérez, L. Guitián y P. Ramil (eds.): La evolución del paisaje en las montañas del entorno de los Caminos Jacobeos. Xunta de Galicia. Santiago de Compostela: 25-60.

Rose, A.W.; HAWkes, H.E. y WeB, J.S. (1979): Geochemistry in mineral exploration. Academic Press Inc. London. $2^{\text {nd }}$ Edition.

SCHÜTZ, L. y RAHN, K.A. (1982): "Trace elements concentrations in erodible soils". Atmospheric Environment, 16: 171-176.

Sнотук, W. (1996): "Peat bog archives of atmospheric metal deposition: geochemical evaluation of peat profiles, natural variations in metal concentrations and metal erichment factors". Environmental Review, 4: 149-183. 